\title{
Universidade Federal do Rio Grande do Sul - UFRGS
}

TÍTULO: Atividade Colonizadora de Basidiomycetes Xilófagos na Presença da Flora Fúngica do Solo, "in vitro".

AUTOR: Soraia Girardi Bruermam

DATA: 02 de junho de 1989

LOCAL: Departamento de Botânica, Universidade Federal do Rio Grande do Sul

NÍVEL: Mestrado

BANCA EXAMINADORA: Rosa R. Guerrero (orientadora)

Lina Bertucci (Facultad Humanidads y Ciências Uruguai)

Adauto Ivo Milanez (Instituto de Botânica de São Paulo)

Maria Luisa Lorschaitter ((UFRGS)

Luis Rios de Moura Baptista (UFRGS)

Norma Wurdig (UFRGS)

RESUMO: Busca-se avaliar a capacidade colonizadora de quatro Basidiomycetes xilófagos, quando incubados na presença da flora fúngica do solo. Os Basidiomycetes utilizados foram: Dacryopinax spathularia (Schw.) Martin, Phaeocoriolellus trabeus (Pers. ex Fr.) Kotl. \& Pouz., Pycnoporus sanguineus (Fr.) Murril e Tyromyces palustris (Berk. \& Curt.) Murril. Destes, $P$. sanguineus causa podridāo branca e os três restantes são responsáveis pelo apodrecimento marrom da madeira. $\mathrm{O}$ estudo da colonizaçāo foi repetido em cada estação do ano e a flora lignofílica dos blocos de madeira identificada. A partir dos fragmentos de madeira extraídos do interior dos blocos-inóculos, observou-se o número de colônias recuperadas de cada Basidiomycete. Dos fragmentos retirados da parte interna dos blocos estéreis, incubados na presença ou nāo de Basidiomycetes, determinou-se o número de colônias das diferentes espécies que constituem a micoflora colonizadora. Com base nestes dados calculou-se o índice de diversidade e similaridade para cada comunidade lignofílica. $\mathrm{O}$ teste $\mathrm{t}$ foi aplicado para detectar as possíveis diferenças estatisticamente significativas. Também analisou-se a ausência de colonizaçāo pelos Basidiomycetes e suas possíveis causas, a influência da sazonalidade sobre as comunidades fúngicas colonizadoras dos blocos de madeira e a inexistência de correlaçāo entre o tipo nutricional de Basidiomycete e a flora fúngica.

TÍTULO: Efeitos Alelopáticos de Mimosa bimucronata (DC.) O. Kunzte (Maricá)

AUTOR: Ubiratā Soares Jacobi

DATA: 10 de agosto de 1989

LOCAL: Departamento de Botânica - Universidade Federal do Rio Grande do Sul

NÍVEL: Mestrado

BANCA EXAMINADORA: Alfredo Gui Ferreira (orientador)

Eloir Schenkel (UFRGS)

Maria E. A. Aquila (UFRGS)

RESUMO: Foram realizados experimentos em laboratório testando os efeitos inibidores de fragmentos de folhas, frutos e extratos aquosos de folhas sobre a germinaçāo, tempo de germinạção e crescimento da radícula de oito espécies cultivadas (alface, arroz, cenoura, 
chicória, couve, pepino, repolho e tomate). Os extratos aquosos de folhas foram preparados com material coletado nas quatro estaçōes do ano em três concentraçōes, 1:8, 1:4 e 1:2. Os fragmentos de folhas frescas e secas inibiram significativamente a germinação e o crescimento das radículas das plantas testadas. Os fragmentos de frutos verdes inibiram significativamente apenas o crescimento das radículas e os fragmentos de frutos maduros não induzem inibiçāo. Extratos obtidos no inverno (folhas velhas) indicaram maior concentraçāo de inibidores do que os de outras estaçōes. Nos extratos obtidos na primavera (folhas jovens) o efeito inibitório foi muito baixo. Mimosina e 3-hidroxi-4 $(1 \mathrm{H})$ - piridona parecem ser as substâncias inibidoras da germinaçāo e crescimento presentes em folhas de Mimosa bimucronata.

TÍTULO: Myxomycetes do Morro Santana (Porto Alegre - Rio Grande do Sul)

AUTOR: Cesar Luiz Mendes Rodrigues

DATA: 23 de outubro de 1989

LOCAL: Departamento de Botânica, Universidade Federal do Rio Grande do Sul

NÍVEL: Mestrado

BANCA EXAMINADORA: Rosa Trinidad Guerrero (orientadora)

Alaides Ribeiro Teixeira (IBSP)

Laise de Holanda Cavalcanti andrade (UFPe)

Hilda Maria Longhi-Wagner (UFRGS)

RESUMO: Este trabalho consiste num levantamento taxonômico levado a efeito no Morro Santana, Porto Alegre, Rio Grande do Sul, no qual foram identificadas 34 espécies pertencentes a 20 gêneros e 6 famílias da classe Myxomycetes. A família mais representada foi Physaraceae (6 gêneros, 11 espécies), seguida por Trichiaceae (4 gêneros, 9 espécies), Didymiaceae ( 3 gêneros, 5 espécies), Stemonitaceae ( 3 gêneros, 5 espécies), Enteridiaceae ( 3 gêneros, 3 espécies) e Ceratiomyxaceae (1 gênero, 1 espécie). São feitas descriçōes, comentários e ilustraçōes para cada espécie. $O$ trabalho inclui uma chave para identificaçāo dos taxa encontrados. Todas as espécies sāo referências novas para o Rio Grande do Sul.

TÍTULO: Revisāo Taxonômica do Gênero Petunia A. L. Jussieu no Rio Grande do Sul, Brasil.

AUTOR: Joāo Renato Stehmann

DATA: $\quad 23$ de outubro de 1989

LOCAL: Departamento de Botânica, Universidade Federal do Rio Grande do Sul

NÍVEL: Mestrado

BANCA EXAMINADORA: Bruno Edgar Irgang (orientador)

Armando F. Hunziker (Córdoba - Argentina)

Lucia F. de Cavalho (Jadim Botânico)

Lilian Auler Mentz (UFRGS)

Luis Rios de Moura Baptista (UFRGS)

RESUMO: O gênero Petunia Jussieu (Solanaceae) é revisado para o Rio Grande do Sul, Brasil. Quinze grupos taxonômicos sāo reconhecidos: $P$. axillaris (Lam.) B.S.P., P. caesia Sendtner /P. sellowiana Sendtner, $P$. excellens $\mathrm{R}$.E. Fries /P. variabilis $\mathrm{R}$.E. Fries, $P$. hetero- 
phylla Sendtner, $P$. humilis R.E. Fries, $P$. integrifolia (Hook.) Schinz et Thellung, $P$. aff. linearis (Hook.) Paxton, P. linoides Sendtner, P. ovalifolia J. Miers, P. parviflora Jussieu, P. pygmaea R.E. Fries, $P$. regnellii R.E. Fries, $P$. tetragonifolia Stehmann e $P$. thymifolia (St.-Hil.) Sendtner. Descriçōes Ilustraçōes, observaçōes fenológicas, habitat, distribuiçāo geográfica e uma chave analítica para identificaçāo dos taxa são apresentadas. A variabilidade e o potencial diagnóstico dos caracteres taxonômicos analisados são discutidos.

TíTULO: Algas Macroscópicas (Chlorophyta, Phaeophyta, Rhodophyta) da Regiāo de Tramandaí e Imbé, RS, Brasil.

AUTOR: Joāo Fernando Prado

DATA: 21 de novembro de 1989

LOCAL: Departamento de Botânica, Universidade Federal do Rio Grande do Sul

NÍVEL: Mestrado

BANCA EXAMINADORA: Luis Rios de Moura Baptista (orientador)

Eurico Cabral de Oliveira Filho (USP)

Marilza Cordeiro Marino (IBSP)

Maria Luiza Lorscheitter (UFRGS)

Eny Correa Vianna (UFRGS)

RESUMO: Sāo caracterizadas as 22 espécies de algas macroscópicas (10 clorófitos, 5 feófitos e 7 rodófitos) encontradas na regiāo de Tramandaí e Imbé com descriçōes, ilustraçōes e chaves artificiais para a identificaçāo. Apresentam-se listas das espécies da regiāo e tabelas de comparação entre os três pontos (Torres, Tramandaí-Imbé, Rio Grande) do litoral sul-riograndense onde se encontram algas macroscópicas, e da composiçāoda flora algal macroscópica da costa do Estado do Rio Grande do Sul. É apresentada a relaçāo total de espécies na costa do Estado.

TÍTULO: Aphyllophorales Poliporóides (Basidiomycetes) do Parque Nacional de Aparados da Serra, RS.

AUTOR: Rosa Mara Borges da Silveira

DATA: $\quad 01$ de agosto de 1990

LOCAL: Departamento de Botânica, Unirversidade Federal do Rio Grande do Sul

NÍVEL: Mestrado

BANCA EXAMINADORA: Rosa Trinidad Guerrero (orientadora)

Jorge Wright (Universidade de Buenos Aires)

Mario Rajhenberg (Universidade de Buenos Aires)

Maria Henriqueta Honrich (UFRGS)

Vera Lucia Nozari Susin (FURG)

RESUMO: No levantamento taxonômico dos Aphyllophorales poliporóides do Parque Nacional de Aparados da Serra, Rio Grande do Sul foram identificados 38 espécies pertencentes a 23 gêneros e 4 famílias. A família melhor representada foi Polyporaceae (18 gêneros, 30 espécies), seguida por Hymenochaetaceae (2 gêneros, 5 espécies), 
Ganodermataceae ( 2 gêneros, 2 espécies) e Corticiaceae (1 gênero, 1 espécie). As espécies Datronia mollis (Somf. ex Fr.) Donk e Junghuhnia nitida (Fr.) Ryv. sāo citaçōes novas para o Brasil. Tyromyces Ieucomallus (Berk. \& Curt.) Murr. é citada pela primeira vez para o Rio Grande do Sul. Sāo apresentadas chaves para identificaçāo, descriçōes, observaçōes e ilustraçōes das espécies estudadas.

TÍTULO: Ilex paraguariensis St. Hil.: Endosperma e Embriāo Durante a Embriogênese Tardia

AUTOR: Eliane Diefenthaler Henser

DATA: 14 de setembro de 1990

LOCAL: Departamento de Botânica, Universidade Federal do Rio Grande do Sul

NÍVEL: Mestrado

BANCA EXAMINADORA: Alfredo Gui ferreira (orientador)

Teresia Strehl (Fundaçāo zoobotânica -RS)

Paulo Luiz de Oliveira (UFRGS)

Regina Termignoni (UFRGS)

Elga Winge (UFRGS)

RESUMO: Sementes de Ilexparaguariensis St. Hil. (erva-mate) necessitam, “in situ”, de um longo período para germinar - 6 a 8 meses - e a percentagem de germinação é muito baixa. Quando os embriōes são excisados e cultivados "in vitro", a embriogênese pode completarse em duas semanas. As causas quanto a esses aspectos do desenvolvimento embrionário tardio ainda nāo entāo bem esclarecidas. A interrupção da embriogênese "in vivo" deve-se, provavelmente, à imaturidade do embriāo. $\mathrm{O}$ baixo índice germinativo pode estar sendo causado por degeneração prematura do suspensor, em alguma das fases desse desenvolvimento. Visando contribuir para a elucidaçāo desse problema, foram feitos estudos morfoanatômicos - tanto em material fresco como em material fixado nas diversas fases do desenvolvimento embrionário, em pirenos de Ilex paraguariensis, cultivados a campo durante doze meses. Lotes foram coletados em intervalos de aproximadamente vinte dias e analisados, permitindo a constatação de mudanças estruturais tais como, formaçāo de cotilédones, aumento gradativo do eixo embrionário, diferenciação de tecidos e espessamento helicoidal de elementos de conduçāo. Pôde-se observar, particularmente, a presença do suspensor, órgāo identificável em estrutura embrionária, durante as diversas fases da embriogênese, em estágio inicial, como também em estágios mais avançados do desenvolvimento. Testes histoquímicos permitiram verificar a natureza lipoprotéica das reservas do endosperma. Estas reservas, em estágios mais avançados de desenvolvimento embrionário, apresentam-se modificadas, na regiāo próxima ao embriāo, mostrando a ocorrência de utilização das mesmas.

TÍTULO: O Gênero Hyptis Jacq. (Labiatae) no Rio Grande do Sul

AUTOR: Sergio Augusto de Loreto Bordignon

DATA: 27 de dezembro de 1990

LOCAL: Departamento de Botânica, Universidade Federal do Rio Grande do Sul NÍVEL: Mestrado 
BANCA EXAMINADORA: Maria Luiza Porto (orientadora)

Hilda Maria Longhi-Wagner (UFRGS)

Raymond Harley (Royal Botanic Gardens - KEW)

Bruno Edgar Irgang (UFRGS)

RESUMO: Foi realizado olevantamento taxonômico das espécies de Hyptis Jacq.(Labiatae), que ocorrem no estado do Rio Grande do Sul. É apresentada uma chave para identificação das espécies e, para cada espécie, descriçāo, comentários, ilustraçōes e mapa de distribuiçāo geográfica do material examinado. O estudo taxonômico foi efetuado através da análise de material coletado durante várias excursōes realizadas durante os anos de 1985-1989 e de revisōes de exsicatas em diversos herbários do sul do Brasil. Até o momento foram encontradas, para o Rio Grande doSul, 16 espécies e 2 subespécies, distribuídas em 7 secçōes, sendo descrita a espécie nova Hyptis tetracephala. É citada pela primeira vez para o Estado Hyptis elegans (Briq.) Briq. ex Mich.

TÍTULO: Cultura de Embrião de Erva-Mate (Ilex paraguariensis St. Hil.) "in vitro".

AUTOR: Geraldo Gonçalves Cunha

DATA: 29 de janeiro de 1991

LOCAL: Departamento de Botânica, Universidade Federal do Rio Grande do Sul

NÍVEL: Mestrado

BANCA EXAMINADORA: Alfredo Gui Ferreira (orientador)

Helga Winge (UFRGS)

Jorge Ernesto de Araujo Mariath (UFRGS)

Luis Mronginski (Corrients - Argentina)

RESUMO: Ilex paraguariensis St. Hil. (erva-mate) tem grande importância econômica e sócio-cultural, principalmente, no sul do Brasil, Uruguai, Argentina e Paraguai, devido à sua utilização na preparação de uma infusão feita com suas folhas, conhecida por chimarrāo. Apesar de sua intensa exploração econômica, poucos estudos têm sido feitos sobre a biologia da erva-mate, o que tem prejudicado a expansāo de sua produçāo, mesmo com uma demanda sempre crescente. As sementes de Ilex para guariensis têm baixa germinabilidade, necessitando de um longo período de estratificaçāo (seis a oito meses) e mais dois ou três meses para a geiminaçāo. Este procedimento é utilizado para superar a dormência destas sementes, que ocorre devido à imaturidade embrionária comum no gênero Ilex. Este trabalho foi realizado com o objetivo de encontrar um meio de superar os impedimentos germinativos da erva-mate, utilizando-se a técnica de cultura de embriōes "in vitro", contribuindo-se, assim, para o desenvolvimento de uma nova tecnologia para produção de mudas dessa espécie. Foi utilizado meio básico de Linsmaier \& Skoog, testando-se variaçōes de seus constituintes e de fatores físicos, como $\mathrm{pH}$, luz e temperatura. Foi, também, testado o efeito de diferentes concentraçōes de sacarose, a influência da adição do endosperma ao meio de cultura, o cultivo em meio sólido ou líquido e a influência da fase de desenvolvimento do fruto no momento da excisāo do embriāo. Constatou-se que os embriōes de I. paraguariensis superaram a sua dormência quando cultivados "in vitro" e que as melhores condiçōes de cultivo sāo em meio líquido, com $1,5 \%$ de sacarose, $\mathrm{pH}$ em torno de 5,5, no escuro, a $25^{\circ} \mathrm{C}$. Constatou-se, ainda, que existem diferenças entre as populaçōes amostradas (Veranópolis e Venâncio Aires) quanto à relação entre a coloração do fruto e o desenvolvimento dos embriōes "in vitro", sendo 
que os embriōes de frutos vermelhos e vináceos da amostra de Venâncio Aires têm um crescimento menor que os embriōes de frutos verdes e brancos, enquanto que na amostra de Veranópolis nāo existe esta diferença. Outra diferença entre as duas populaçōes é a influência da luz sobre o crescimento dos embriōes oriundos de frutos brancos, que na populaçāo de Venâncio Aires não sofreram influência do espectro luminoso, enquanto que os embriōes oriundos de frutos das demais fases, de ambas as populaçōes, tiveram o seu crescimento reduzido em presença de luz.

TÍTULO: Levantamento da Flora Rupestre do Morro do Cabrito e Morro Sapucaia, Rio Grande do Sul, Brasil.

AUTOR: Irene Fernandes

DATA: 01 de abril de 1991

LOCAL: Departamento de Botânica, Universidade Federal do Rio Grande do Sul

NÍVEL: Mestrado

BANCA EXAMINADORA: Luis Rios de Moura Baptista (orientador)

Maria Luiza Porto (UFRGS)

Bruno Edgar Irgang (UFRGS)

Jorge Pedro Carauta (FEEMA)

RESUMO: Realizou-se o levantamento da flora vascular rupestre de dois morros testemunhos areníticos do Rio Grande do Sul, Brasil. Este trabalho inclui dados sobre formas de vida, sociabilidade e abundância, habitats ocupados, distribuição espacial nos locais de estudo e distribuição geográfica das espécies encontradas, além de aspectos gerais da vegetação dos morros. Foram encontradas 106 espécies de plantas vasculares rupestres, sendo 34 de pteridófitas e 72 de angiospermas. As formas de vida predominantes entre as espécies rupestres encontradas são os caméfitos reptantes ou decumbentes e os nanoe microfanerófitos perenifolios. Em muitos casos, observou-se grande variaçāo morfológica em plantas da mesma espécie que crescem em condiçōes ecológicas distintas.

TíTULO: Flora Espermatófita do Estado de Augusto Cesar, Rio Uruguai - Marcelino Ramos, RS - Concórdia, SC.

AUTOR: Elisabete Maria Zanin

DATA: 10 de julho de 1991

LOCAL: Departamento de Botânica, Universidade Federal do Rio Grande do Sul

NÍVEL: Mestrado

BANCA EXAMINADORA: Bruno Edgar Irgang (orientador)

Ca:los Alfredo Joly (UNICAMP)

Luis Rios de Moura Baptista (UFRGS)

Maria Luiza Porto (UFRGS)

RESUMO: O presente trabalho constitui-se no estudo da flora espermatófita do Estreito de Augusto César, situado no rio Uruguai, entre a cidade de Marcelino Ramos, RS e a cidade de Concórdia, SC. A área de estudo inicia a $51^{\circ} 57^{\prime} 25^{\prime \prime} \mathrm{O}$ e termina a $52^{\circ} 00^{\prime} 03^{\prime \prime} \mathrm{O}$. A latitude do início do canal é $27^{\circ} 24^{\prime} 51^{\prime}$ 'S e do término é $27^{\circ} 24^{\prime} 13^{\prime}$ 'S. Foram encontradas 202 espécies pertencentes a 58 famílias, das quais uma espécie constitui citação nova para o Rio Grande do Sul. Apresentam-se breves descriçōes das espécies, incluindo a caracterizaçāo das mesmas, considerando-se as formas biológicas, observaçōes ecológicas e fenológicas. Inclui- 
se, ainda, uma descrição da distribuição das espécies na área com o respectivo diagrama de perfil e fotos ilustrativas.

TÍTULO: O Gênero Stipa L. (Gramineae - Stipeae) no Brasil

AUTOR: Ana Zanin

DATA: 19 de agosto de 1991

LOCAL: Departamento de Botânica, Universidade Federal do Rio Grande do Sul

NÍVEL: Mestrado

BANCA EXAMINADORA: Hilda Maria Longhi-Wagner (orientadora)

Ilsi Iob Boldrini (UFRGS)

Ana Maria Giulietti (USP)

José Francisco Montenegro Valles(CENARGEN/EMBRAPA)

RESUMO: Este trabalho constitui-se em um levantamento taxonômico das espécies de Stipa L. (Gramineae - Stipeae) ocorrentes no Brasil. Com base em coletas, revisāo de herbários e observaçōes de populaçōes no campo, foi confirmada a ocorrência de 25 espécies e duas variedades. S. quinqueciliata (Roseng. \& Izag.) A. Zanin \& Izag. nov.comb., ined., S. setigera var. longiaristata (Arech.) Roseng. e $S$. torquata Speg. são citaçōes novas para o Brasil. $S$. brasiliensis, $S$. planaltina, $S$. rhizomata $S$. vallsii sāo espécies novas para a ciência, descritas durante a realização deste trabalho. $O$ trabalho inclui chaves analíticas para as espécies e variedades, além de descriçōes e ilustraçōes das mesmas. Também fornece dados sobre habitat, fenologia e distribuiçāo geográfica de cada táxon estudado.

TÍTULO: Palinologia de uma Turfeira do Parque Nacional de Aparados da Serra, Planalto Leste do Rio Grande do Sul

AUTOR: Lionel Roth

DATA: 23 de setembro de 1991

LOCAL: Departamento de Botânica, Universidade Federal do Rio Grande do Sul

NÍVEL: Mestrado

BANCA EXAMINADORA: Maria Luisa Lorscheitter (orientadora)

Maria Lea Salgado-Laboriau (UnB)

Eduardo Juan Romero (Universidade de Buenos Aires)

Alfredo Gui Ferreira (UFRGS)

Jorge Luiz Waechter (UFRGS)

RESUMO: Realizou-se o estudo palinológico de uma turfeira do Parque Nacional de Aparados da Serra, Planalto Leste, Rio Grande do Sul, Brasil, com objetivo de obter dados sobre câmbios vegetacionais e climáticos do Quaternário Superior da regiāo. Foi feita análise qualitativa e quantitativa de palinomorfos contidos em 37 amostras, coletadas da base ao topo do perfil sedimentar escolhido. $\mathrm{Na}$ análise qualitativa foram descritos 100 palinomorfos encontrados: fungos (13), algas (6), briófitos (4), pteridófitos (10), gimnospermas (1), angiosperamas (57), além de outros palinomorfos, não referentes a esses grupos principais. $\mathrm{Na}$ análise quantitativa os palinomorfos encontrados foram trabalhados com vistas às interpretaçōes paleoambientais. Dois métodos foram utilizados: método de porcentagem e método de concentração polínica. Análises adicionais também foram levadas em consideraçōes, relacionadas à litologia, geoquímica e datação radiométrica de sedimentos, além do estudo de chuva polínica atual. Os resultados obtidos permitiram detectar câmbios vegetaci- 
onais e climáticos significativos junto ao limite Pleistoceno-Holoceno e oscilaçōes de menor amplitude durante o Holoceno. Ficou evidenciado o avanço gradual da mata de Araucaria sobre o campo, durante os últimos milênios.

TÍTULO: O Gênero Piptochaetium Presl (Gramineal-Stipeae) no Brasil

AUTOR: Jaime Mujica Salles

DATA: 28 de setembro de 1991

LOCAL: Departamento de Botânica, Universidade Federal do Rio Grande do Sul

NÍVEL: Mestrado

BANCA EXAMINADORA: Ilsi Iob Boldrini (UFRGS)

José Francisco Montenegro Valles(CENARGEN/EMBRAPA)

Primavera Izaguirre de Artucio (Faculdade de Agronomiade Montevideo)

RESUMO: Foi feito olevantamento taxonômico das espécies do gênero Piptochaetium Presl (Graminese - Stipeae) presentes no Brasil, como uma contribuição ao conhecimento ecológico dos campos naturais. Com base no estudo de material coletado para este trabalho, observaçōes das populaçōes no campo e revisāo da literatura e de herbários, foram reconhecidos 15 taxa, 9 espécies e 6 variedades. É proposta a sinonimizaçāo de $P$. confusum Parodi sob $P$. ruprechtianum Desv. Uma nova espécie para a ciência, $P$. palustre Mujica \& Longhi-Wagner, é apresentada. Além disto, P. panicoides var. subpapillosum (Hack.) Petetin e $P$. uruguense var. microcarpum Parodi constituem-se em novas citaçōes para o Brasil. São fornecidas chaves analíticas para as espécies e variedades, descriçōes e ilustraçōes das mesmas bem como dados de distribuição geográfica, habitat e fenologia.

TÍTULO: Anatomia dos Orgāos Vegetativos de Chenopodium retusum Juss. ex Moq. (Chenopodiaceae).

AUTOR: Francisco Antonio Silva Filho

DATA: 21 de outubro de 1991

LOCAL: Departamento de Botânica, Universidade Federal do Rio Grande do Sul

NÍVEL: Mestrado

BANCA EXAMINADORA: Paulo Luiz de Oliveira (orientador)

Jorge Ernesto de Araujo Mariath (UFRGS)

Jane Elizabeth Kraus (USP)

Cecília Beltrati (UNESP)

Teresia Strehl (Fundação zoobotânica - RS)

RESUMO: Chenopodium retusum Juss. ex Moq. é uma erva perene da vegetaçāo costeira arenosa do Sul do Brasil, Uruguai e Argentina. Além das técnicas usuais para estudos anatômicos, foram empregados diversos testes histoquímicos para determinaçāo e localizaçāo de cutina, celulose, lignina, lipídios, polissacarídeos totais, proteinas totais, amido e tanino em todos os tecidos das folhas, caules e raízes. As raízes foram estudadas em estruturas primária e secundária, desde regiōes jovens até adultas. Seu crescimento secundário é caracterizado pela sucessāo de vários câmbios supernumerários extrafasciculares concêntricos, com fraca lignificação de seus tecidos. Os caules foram examinados em estrutura primária, crescimento secundário, filotaxia, traço e lacuna foliares, desde o ápice a té o $54^{\circ}$ nó em seqüência, e a partir daí a intervalos de $10 \mathrm{em} 10$ nós, até o $94^{\circ}$ nó. Folhas jovens e adultas 
foram examinades em cinco regiōes: proximal, supra-proximal, mediana, sub-distal e distal, sendo caracterizada a epiderme e seus anexos, mesofilo e nervura mediana. Aspectos anatômicos dos três órgãos são discutidos com ênfase nas características adaptativas ao ambiente arenoso-salino e no crescimento secundário "anômalo" de raiz e caule.

TíTULO: Urticaceae do Rio Grande do Sul

AUTOR: Paulo Brack

DATA: $\quad 21$ de outubro de 1991

LOCAL: Departamento de Botânica, Universidade Federal do Rio Grande do Sul

NÍVEL: Mestrado

BANCA EXAMINADORA: Bruno Irgang (orientador)

Jorge Pedro Carauta (FEEMA)

Maria Luisa Lorscheitter (UFRGS)

Maria Luiza Porto (UFRGS)

RESUMO: Este trabalho tem como objetivo realizar o levantamento taxonômico da família Urticaceae, dentro da linha de pesquisa sobre a flora do Rio Grande do Sul (Brasil), enfocando-se as espécies nativas ou de ocorrência espontânea no Estado.

A taxonomia foi estabelecida a partir de análises de coletas e exsicatas recolhidas em diversos herbários do Brasil e alguns países vizinhos. Foram encontrados, até o presente, para o Rio Grande do Sul, 6 gêneros e 16 espécies, sendo descrita a espécie nova Pilea aparadensis Prack. É referido pela primeira vez para o Brasil Phenax uliginosus Wedd, e pela primeira vez para o Estado, $P$. organensis Glaz, e Pilea hilariana Wedd. Apresentam-se chaves para gêneros de Urticaceae e para cada espécie, descriçāo, comentários, ilustraçōes e mapas de distribuiçāo de coletas.

TíTULO: Anatomia de Raízes de Orquídeas Terrestres Naturais no Morro Santana, Porto Alegre, RS.

AUTOR: Mara Lisiane Tissot

DATA: $\quad 28$ de outubro de 1991

BANCA EXAMINADORA: Teresia Strehl ( Funcaçāo Zoobotânica - RS)

Maria Luiza Porto (UFRGS)

Jorge Ernesto de Araújo Mariath (UFRGS)

RESUMO: Investigou-se a anatomia das raízes adventícias de seis espécies de orquídeas terrestres nativas no Morro Santana (Porto Alegre, RS); distribuídas nas quatro sub-famílias representadas no Rio Grande do Sul:Mesadenellacuspidata, Prescottia stachyodes, Habenaria josephensis, Liparis nervosa, Epidendrum fulgens e Warrea warreana. As seis espécies mostraram ter ápice radical do tipo fechado, com três grupos de células iniciais: do cilindro vascular, do córtex-epiderme e da coifa. Em todos os casos a protoderme dá origem a um velame uni a pluri-estratificado. Quatro das seis espécies estudadas possuem tilossomas nas paredes internas das células do velame em contato com a exoderme. O parênquima cortical em $M$. cuspidata e $L$. nervosa mostra-se esclerificado nas partes maduras da raiz. A endoderme apresenta estria de Caspary. Espessamentos nas paredes das células da endoderme opostas ao floema ocorrem em todas as espécies estudadas, exceto em $P$. stachyodes e H. josephensis. Em M. cuspidata e E. fulgens ocorre espessamento nas paredes também das células-de-passagem da endoderme, nas partes mais velhas da raiz. $O$ ciclindro vascular é 
delimitado por um periciclo uniestratificado, cujas paredes podem apresentar espessamento; este segue o padrāo da endoderme. As raízes das espécies examinadas sāo poliarcas, com número variado de grupos vasculares, distribuídos radialmente em torno de uma medula parenquimática. Foram reconhecidos dois padrōes de diferenciaçāo vascular: 1) poucos grupos vasculares iniciais dāo origem aos demais, por separaçāo das células procambiais ou de elementos condutores em diferenciaçāo, em $M$. cuspidata e $P$. stachyodes; 2) todos os grupos casculares se formam a partir de grupos celulares precursores definidos anteriormente, nas demais espécies. As células do parênquima vascular apresentam espessamento de paredes, exceto em $P$. stachyodes e $H$. josephensis.

TÍTULO: Estudo Taxonômico do Gênero Mikania Willd. (Asteraceae) secçōes Globosae e Thyrsigerae no Rio Grande do Sul

AUTOR: Mara Rejane Ritter

DATA: 1990

LOCAL: Departamento de Botânica, Universidade Federal do Rio Grande do Sul

NÍVEL: Mestrado

BANCA EXAMINADORA: Luis Rios de Moura Baptista (orientador)

Nelson Ivo Matzenbacher (orientador)

RESUMO: Este trabalho é um inventário do gênero Mikania Willd. (Asteraceae), secçōes Globosae e Thyrsigerae, no estado do Rio Grande do Sul. Sāo feitas consideraçōes sobre a família, o gênero e secçōes propostas pelos autores. É apresentada uma descrição do gênero, chaves para identificaçāo das secçōes e das espécies de Mikania no Rio Grande do Sul. Foram encontradas duas espécies de Mikania da secção Globosae e 16 espécies da secpāo Thyrsigerae para o Estado. Para cada espécie sāo apresentadas descriçōes, observaçōes, distribuiçāo geográfica, fenologia, ilustraçōes e mapa de distribuiçāo das coletas no Rio Grande do Sul. Constatou-se a ocorrência de cinco novas citaçōes para a Flora do Rio Grande do Sul.

\section{Universidade Federal Rural de Pernambuco - UFRPE}

TÍTULO: Estimativas de Parâmetros de Alguns Caracteres Agricolas da Cana-de-Açúcar (Saccharum spp).

AUTOR: Cláudio José Calábria Cavalcanti

DATA: 29 de maio de 1990

LOCAL: Universidade Federal Rural de Pernambuco

NÍVEL: Mestrado

BANCA EXAMINADORA: Mário de Andrade Lira (Orientador)

Margarida Agostinho Lemos

Clodoaldo José da Anunciação Filho

Gothardo Marcon

RESUMO: Com o objetivo de se estimar parâmetros genéticos em alguns caracteres de canade-açúcar (Saccharum spp.), foram conduzidos seis experimentos nas quatro principais zonas 